Regazzoni, Pierre-Louis; Marot, Didier; Nguyen, H. H.

Surface Erosion: Erodibility Characterisation and Physical Parameters Effects

Verfügbar unter / Available at:

https://hdl.handle.net/20.500.11970/100245

Vorgeschlagene Zitierweise / Suggested citation:

Regazzoni, Pierre-Louis; Marot, Didier; Nguyen, H. H. (2010): Surface Erosion: Erodibility Characterisation and Physical Parameters Effects. In: Burns, Susan E.; Bhatia, Shobha K.; Avila, Catherine M. C.; Hunt, Beatrice E. (Hg.): Proceedings 5th International Conference on Scour and Erosion (ICSE-5), November 7-10, 2010, San Francisco, USA. Reston, Va.: American Society of Civil Engineers. S. 182-191. 


\title{
Surface Erosion: Erodibility Characterisation and Physical Parameters Effects
}

\author{
P.L. Regazzoni, D. Marot and H.H. Nguyen
}

University of Nantes, Institut GeM, BP 42044606 Saint-Nazaire Cedex, France; email : pierre-louis.regazzoni@etu.univ-nantes.fr, didier.marot@univ-nantes.fr, hong-hai.nguyen@univ-nantes.fr

\begin{abstract}
Erosion is one of the main causes of instabilities within hydraulic earth structures. Two types of erosion can be distinguished: internal erosion and surface erosion. This paper deals with the surface erosion phenomenon and the Jet Erosion Test is used in order to evaluate the erodibility of cohesive soils.

A new energy analysis of the test is developed, linking the expended energy to the erosion phenomenon. The total eroded mass is correlated to the expended fluid energy and a new erosion resistance index is proposed.

The erodibility is evaluated for several natural soil samples which are compacted with the Proctor protocol and which represent a large panel of erosion sensitivity.

Two dissipated hydraulic energy scales appear, and a statistical analysis is carried out which gives a correlation of the erosion resistance index with three physical parameters.
\end{abstract}

\section{INTRODUCTION}

The interaction between water and hydraulic earth structures such as dams, dikes or levees can cause significant damage to these structures. Erosion appears to be one of the main causes of these instabilities (Foster et al., 2000). Two types of erosion can be distinguished: internal erosion which takes place inside the soil matrix, and surface erosion which occurs at the soil/water interface, or at a material interface soil matrix (for example between two different soils).

With the objective to characterize the surface erosion sensitivity of fine soils, an experimental investigation is carried out with the Jet Erosion Test. Twelve natural soil samples which represent a large panel of erosion sensitivity are compacted with the Proctor protocol.

The test interpretation is performed with a new method based on the dissipated hydraulic energy and the eroded mass. The classification of erosion sensibility is defined by a new erosion resistance index.

A multivariate statistical analysis is performed in order to estimate the erosion resistance index as a function of several variables. The results from this study allow the number of variables for the description of the erosion resistance index measurements to be optimised and reduced. 


\section{EROSION DEVICE AND EROSION RESISTANCE INDEX}

\section{Principle of Jet Erosion Test}

The Jet Erosion Test was developed by Hanson and Simon (2001). A hydraulic jet is created by setting a head loss on a diaphragm (see Figure 1). A point gage is adjusted to close off the nozzle, and also allows one to measure the depth of scour below the nozzle. A submergence tank holds the sample. The jet tube is mounted to the submergence tank cover so that the height of the nozzle above the soil surface can be adjusted to different heights prior to the start of a test. The jet tube and cover can also be mounted to a heavy-duty field tank for in situ measurements.

The collected data during the test at specific times include: the depth of scour $\mathrm{J}$ measured from a reference level and the head applied to the nozzle, $\Delta H$. Data are recorded at intervals chosen by the operator, depending on the erosion rate. Typical intervals range from $15 \mathrm{~s}$ to $30 \mathrm{~min}$, with total test times of 2 hours or less (Hanson and Cook, 2004). With these data, it is possible to relate the hydraulic conditions at interface to the erosion rate at a time $t$.

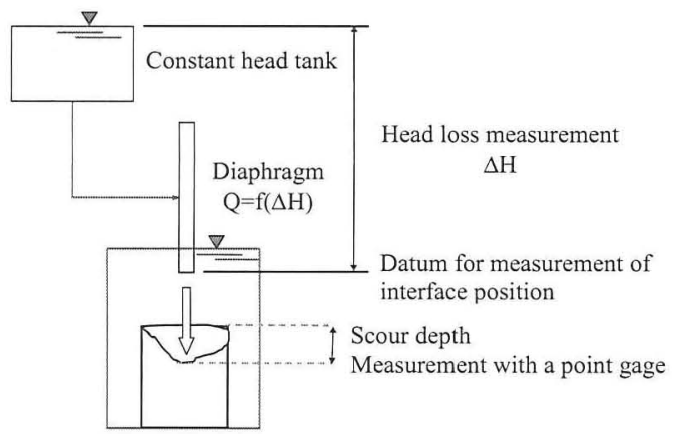

Figure 1. Jet Erosion Test principle.

\section{Water velocity}

With the objective to evaluate the water velocity in the area of the impact, two zones are defined (see Figure 2). In the zone I (altitude $Z<\mathrm{J}_{\mathrm{P}}=6.2 \mathrm{~d}_{0}$, where $\mathrm{d}_{0}$ : diameter of the jet at the exit), the water velocity on the axis is constant and equal to exit velocity (axial velocity $\mathrm{u}=\mathrm{U}_{0}$, radial velocity $\mathrm{v}=0$ ). In the zone II $\left(Z>J_{P}=6.2 d_{0}\right)$ and far from the interface $(Z<0.86-0.9 J)$, the longitudinal velocity $(\mathrm{u})$ on the axis is proportional to the inverse value of the distance between the jet origin and the altitude considered. According to the measurements made by Beltaos and Rajartanam (1974), a coefficient $\left(C_{d} d_{0}\right)$ is introduced in order to obtain a computed velocity in agreement with the measures:

$$
u(0, J)=u(0,0) \frac{C_{d} d_{0}}{Z}
$$


where $\mathrm{u}(0,0)$ is the initial velocity at the jet origin, $\mathrm{d}_{0}$ : diameter of the jet at the exit; $\mathrm{J}$ : distance between soil-water interface and jet origin.

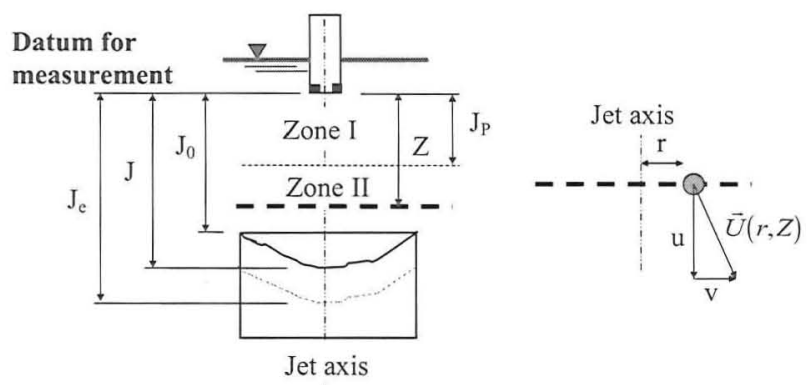

Figure 2. Different measurements during Jet Erosion Test.

In the zone near the interface $(\mathrm{J}>\mathrm{Z}>0.9-0.86 \mathrm{~J})$, the axial velocity $(\mathrm{u}(\mathrm{r}, \mathrm{Z}))$ decreases to 0 to be converted into radial velocity $(v(r, Z))$. Beltaos and Rajaratnam (1974) proposed an expression of the vertical velocity on the jet axis:

$$
\frac{u(r, z)}{u(0, z)}=\exp \left(-0,693\left(\frac{r}{b_{u}}\right)^{2}\right)
$$

with $\mathrm{u}(0, \mathrm{~J})$ : water velocity at the distance $\mathrm{J}$ from the jet origin on the jet axis in the case of a free jet.

$\mathrm{u}(\mathrm{r}, \mathrm{Z})$ : water velocity at a distance $\mathrm{r}$ from the jet axe and a distance $\mathrm{Z}$ from the jet origin in the axial direction

$\rho_{\mathrm{w}}$ : water density

$b_{u}$ : distance from the axis where the water velocity on the axis is divided by two, $b_{u}=0,093\left(J-J_{P}\right)$.

\section{Energy analysis}

Regazzoni (2009) proposed a method of interpretation based on the energy dissipation between the fluid and the soil. The energy equation for the fluid (neglecting the soil phase inside the volume) can be written as:

$$
\begin{aligned}
& \frac{\mathrm{dE}}{\mathrm{dt}}=\frac{\mathrm{d}}{\mathrm{dt}} \iiint \int_{\text {Mass }}\left(e_{\text {int }}+\frac{\mathrm{u}^{2}}{2}+\overrightarrow{\mathrm{g}} \cdot \overrightarrow{\mathrm{x}}\right) \cdot \mathrm{dM}
\end{aligned}
$$

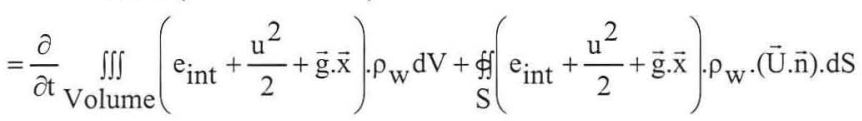


where M: fluid mass, V: fluid volume, $e_{\text {int }}$ internal energy, $S$ : interface between fluid and environment, $\mathrm{n}$ : normal vector of interface, $\mathrm{U}$ : fluid velocity (components: $\mathrm{u}, \mathrm{v}$, w), g: gravity, $\rho_{\mathrm{w}}$ : fluid density, $x$ : coordinates.

Total energy is the sum of the mechanical work $W$ and the energy exchange between the system and the environment $\mathrm{E}_{\text {Ther: }}$ :

$$
\frac{d E}{d t}=\frac{d E_{\text {Ther }}}{d t}+\frac{d W}{d t}
$$

The system can be considered isothermal in time, so internal energy is assumed constant. During the testing time, the system is assumed as adiabatic and the exchange between the system and the environment is neglected $\left(\frac{d_{E_{\text {Ther }}}}{d t}=0\right)$. The assumption of a steady state (locally in time) allows neglecting the unsteady term of the kinetic energy. Finally the equation (3) becomes

$$
\frac{d W}{d t}=\oiint_{S}\left(\frac{u^{2}}{2}+\vec{g} \cdot \vec{x}\right) \rho_{w}(\vec{U} \cdot \vec{n}) \cdot d S
$$

The mechanical work $\mathrm{W}$ is the sum of: work done by pressure, viscous work in the fluid and work by erosion:

$$
\frac{d W_{\text {pressure }}}{d t}+\frac{d W_{\text {viscous in fluid }}}{d t}+\frac{d W_{\text {erosion }}}{d t}=\oint_{S}\left(\frac{u^{2}}{2}+\vec{g} \cdot \vec{x}\right) \cdot \rho_{w} \cdot(\vec{U} \cdot \vec{n}) \cdot d S
$$

Two assumptions are made:

-before and after the impact, pressure is assumed hydrostatic, -the jet deviation is assumed to be the cause of erosion.

The spatial zone concerned by jet deviation is defined by the increasing of radial water velocity. Beltaos and Rajaratnam (1974) noted that radial velocity increases for $r / Z<0.14$. So it is assumed that the energy coming from the jet outside of this area (defined by $\mathrm{r} / \mathrm{Z}<0.14$ ) is dissipated in the fluid.

In the case where $J>J_{P}$, by combination of equations (6) and (2), the energy equation for the fluid can be expressed by:

$$
\frac{d W_{\text {erosion }}}{d t}=2 \pi \int_{0}^{0.14 J} \frac{u^{2}}{2} \rho_{w}(\vec{U} \cdot \vec{n}) r d r=2 \pi \int_{0}^{0.14 J} \rho_{w} \frac{u^{3}(0, J)}{2}\left(\exp \left(-0.693\left(\frac{r}{b_{u}}\right)^{2}\right)\right)^{3} r d r
$$

In the case of $\mathrm{J}<\mathrm{J}_{\mathrm{P}}$, the water velocity is assumed to be constant and equal to the speed at the jet exit. So the energy equation is:

$$
\frac{d}{d t} W_{\text {erosion }}=\pi \rho_{w} u^{3}(0,0)\left(\left(\frac{J_{p}-J}{J_{p}}\right) \frac{d 0}{2}\right)^{2}
$$

To classify the soil according to the erosion, an erosion coefficient is proposed: 


$$
\alpha=\frac{m_{d r y}^{\bullet}}{\frac{d}{d t} W_{\text {erosion }}}
$$

with $m_{d r y}^{\circ}$ : rate of eroded dry mass

By integrating equation (9) over the test duration, the erosion resistance index is built with the erosion energy $\left(E_{\text {erosion }}\right)$ and the eroded dry mass $\left(m_{\text {dry }}\right)$ :

$$
I_{\alpha}=-\log \left(\frac{m_{d r y}}{E_{\text {erosion }}}\right)
$$

\section{EXPERIMENTAL INVESTIGATION}

\section{Soils properties and testing program}

The testing program concerns 12 soils. 8 soils are natural (Regazzoni et al, 2008 ) and 2 soils were created on the basis of natural soils and 2 soils were created using industrial soil materials. The soils are covering a large part of the Atterberg limits diagram (see Figure 3).

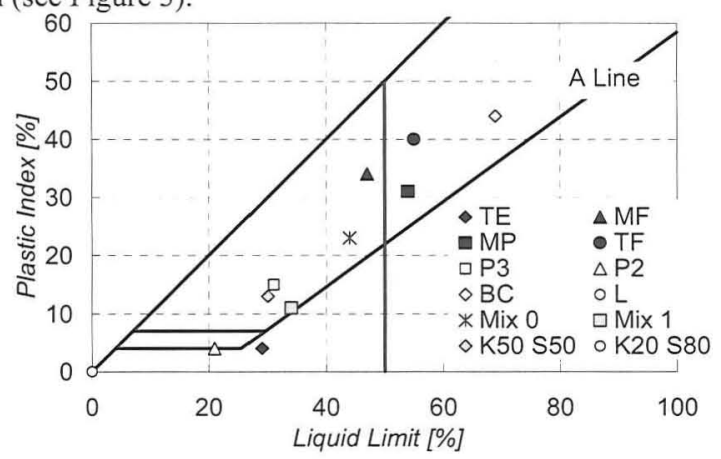

Figure 3. Casagrande diagram.

The optimal dry densities for the Proctor compaction are ranging between $1900 \mathrm{~kg} / \mathrm{m}^{3}$ and $1378 \mathrm{~kg} / \mathrm{m}^{3}$ and the values of optimum water content are between 10 and $24 \%$. A test consists in a compaction with the standard procedure and a Jet Erosion Test.

The preparation of the sample is made according to the following procedures. First the natural soils are prepared, it means: a drying at $65^{\circ} \mathrm{C}$, the crushing and sieving at \#4 A.S.T.M. sieve. For all tested soils, water is added and blended to target optimum water content less $1 \%$ (in conformity with procedure defined by USBR, 1987). The soil is let $36 \mathrm{~h}$ (at least) in a plastic bag. The compaction is made in three layers of 25 blows with a normal Proctor rammer. The sample is let in a plastic bag for 12 hours before test. 


\section{Results of testing}

The tests duration is ranging from $1740 \mathrm{~s}$ to $6300 \mathrm{~s}$. We can distinguish two main categories of soil erodibility: an energy erosion soil higher than 600 Joules (see Figure 4) and a low energy erosion soil (see Figure 5).

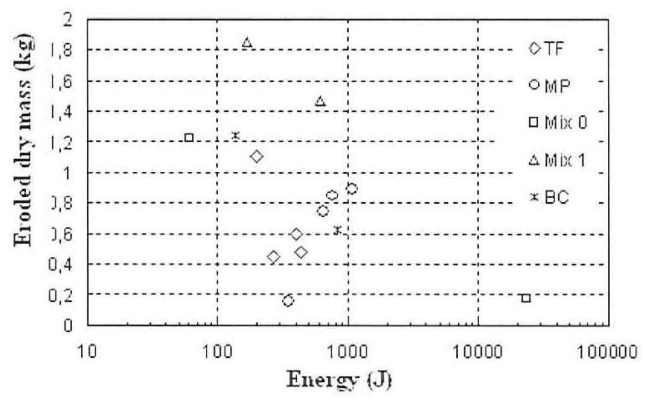

Figure 4. Eroded dry mass vs high energy.

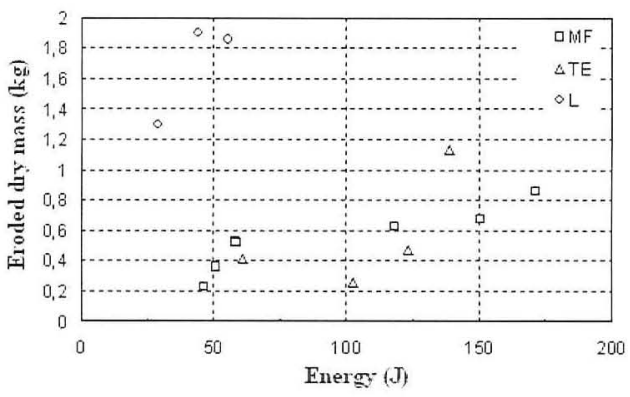

Figure 5. Eroded dry mass vs low energy.

The great difference in erosion sensitivity may be due to the great variability of tested soils.

\section{ESTIMATION OF EROSION RESISTANCE INDEX FROM OTHER SOIL PROPERTIES}

\section{Definition of used parameters}

The used parameters for the statistical analysis try to represent the soil in several characteristics. The first characteristic considers the grain size distribution. The size curve distribution is introduced by considering the clay fraction $\left(\mathrm{F}_{\text {clay }}\right)$ of the soil (size of particles $d \leq 2 \mu \mathrm{m})$, the silts fraction $(2 \mu \mathrm{m}<\mathrm{d} \leq 74 \mu \mathrm{m}$ ), the fine sand fraction $(74 \mu \mathrm{m}<\mathrm{d} \leq 425 \mu \mathrm{m})$ and the coarse sand fraction $(425 \mu \mathrm{m}<\mathrm{d} \leq 4750 \mu \mathrm{m})$. The characterization is completed by the Atterberg limits 
on the soil fraction below $425 \mu \mathrm{m}$. For the water in the soil, the water content, w, and the saturation ratio $S_{\mathrm{r}}$ are considered. To describe the soil structure, the compaction, $\mathrm{c}$, and the dry densities are considered:

$$
c=\frac{\rho_{d}}{\rho_{S}}
$$

where $\rho_{\mathrm{d}}$ : dry density of the soil; $\rho_{\mathrm{s}}$ : solid density. introduced:

To represent the interaction between clay and water, the clay water content is

$$
\mathrm{w}_{\text {clay }}=\frac{\mathrm{w}}{\mathrm{F}_{\text {clay }}}
$$
defined:

Two parameters linking the Atterberg limits to the clay water content are

$$
\begin{aligned}
& \mathrm{w}_{\mathrm{LL}}=\mathrm{LL}-\mathrm{w}_{\text {clay }} \\
& \mathrm{w}_{\mathrm{PL}}=\mathrm{w}_{\text {clay }}-\mathrm{PL}
\end{aligned}
$$

where LL: liquid limit and PL: plastic limit

With the objective to represent the soil water exchange, the surface exchange $\mathrm{S}_{\mathrm{d}}$ is defined by:

$$
S_{d}=6 \sum \frac{1}{d_{50, X i}} p_{i} c
$$

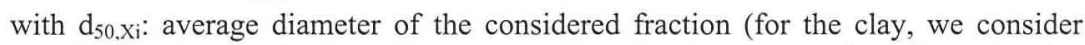
$2 \mu \mathrm{m}$ ); for the sand, the silt, average diameter is computed with grain size distribution; $\mathrm{p}_{\mathrm{i}}$ : percentage in composition of the considered fraction. 


\section{Principle of statistical analysis}

Multivariate analysis allows the full set of variables related to the measurements to be reduced to a subset representing the principal components assuming a linear correlation between the variables. Each parameter is represented in a factor space, and the geometrical representation associates a vector to each parameter. The scalar product of two associated vectors is equal to the correlation coefficient of the two parameters. An automatic classification is used to define all variables according to the most useful factors. Figure 6 shows the variables in first factor plane. The variables list is given below:
$0: \mid \alpha:$ Erosion resistance index.
5: Silt fraction
9: $\log \left(\mathrm{S}_{\mathrm{d}}\right): \log$ arithm of the specific area
1: $\mathrm{w}_{\mathrm{LL}}$
6: Dry density
10: w: Water content
2: $\mathrm{W}_{\mathrm{PL}}$
7: Saturation ratio
11: Fine sand fraction
3: $w_{\text {Argile }}$ : water content of the clay
8: Compaction
12: Coarse sand fraction
4: $\mathrm{F}_{\text {clay: }}$ : Clay fraction

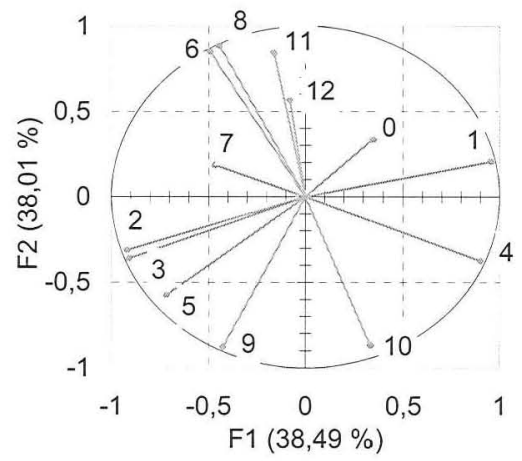

Figure 6. Representation of the variables in first factor plane.

Thus in each plan, the interpretation of parameters is made according to the position from an unit radius circle. Two variables are in linear relationship when their positions are near the unit circle, and very close to each other or diametrically opposite (for example variables $6\left(\rho_{\mathrm{d}}\right), 8(\mathrm{c})$ and $10(\mathrm{w})$ on Figure 6). Two variables are independent when their representations are in quadrature (for example variables 4 $\left(\mathrm{F}_{\text {clay }}\right)$ and $9\left(\log \left(\mathrm{S}_{\mathrm{d}}\right)\right)$ on Figure 6).

Now, we are eliminating the variables which are correlated, or seem meaningless by their redundancy information with other variables.

By leading a new multivariate analysis, three parameters are kept and the correlation with erosion resistance index is:

$$
I_{\alpha}=-0.97+0.47 w_{L L}-0.36 c+5.41 S_{r}
$$


The obtained correlation coefficient $\left(\mathrm{R}^{2}\right)$ between the prediction and the measurement is 0.35 with a number of items $\mathrm{N}=38$. By observing the distribution of the error (cf. Figure 7), it appears that the problematic soils are represented by MF, Mix 0, Mix 1. These soils are dispersive, so now we take into account the dispersive property of the soils.

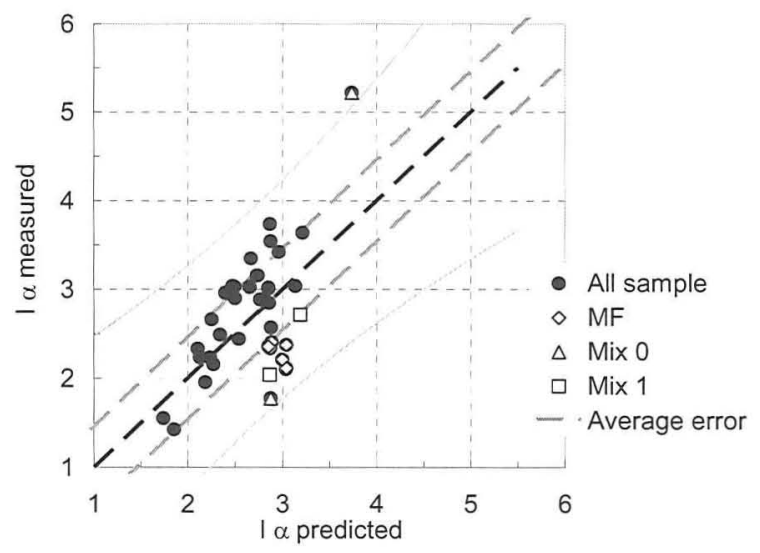

Figure 7. Erosion resistance index, measured values vs predicted values.

A new correlation is defined for non-dispersive soils:

$$
I_{\alpha}=-2.31+0.69 w_{L L}+1.41 c+6.07 S_{r} \quad\left(\mathrm{R}^{2}=0.59, \mathrm{~N}=27\right)
$$

For the dispersive soils the expression of estimated value of $I_{\alpha}$ is:

$$
I_{\alpha}=-1.36+8.69 w_{L L}+2.68 c+2.08 S_{r} \quad\left(\mathrm{R}^{2}=0.81, \mathrm{~N}=11\right)
$$

As a conclusion of this statistical analysis, by distinguishing between dispersive and non-dispersive soils, we identify the main parameters for a soil analysis in relation to the surface erosion phenomenon. Namely: compaction saturation ratio and difference between clay water content and liquid limit.

\section{CONCLUSION}

A Jet Erosion Test device is used in order to characterize the sensitivity to erosion of twelve fine soils which cover a large part of the Atterberg limits diagram. The tested samples are compacted with the standard Proctor procedure at optimum water content less $1 \%$. Study of energy exchanges between fluid and soil leads to propose a new analysis of Jet Erosion Test and a new erosion resistance index is proposed. 
Several physical parameters are determined and a statistical analysis is performed in order to identify the main parameters for a correlation with erosion resistance index.

By distinguishing the dispersive behaviour from non-dispersive behaviour, the multivariate statistical analysis leads to an expression of the erosion resistance index as a function of three physical parameters: compaction, saturation ratio and difference between clay water content and liquid limit. Thus this method allows reducing the number of variables for the description of the erosion sensitivity.

In contrast to the precedent models based on stress, energy model leads to a same classification of soil surface erodibility for two types of apparatus: Jet Erosion Test and Hole Erosion Test (Regazzoni, 2009). Moreover, the analysis based on energy dissipation offers the potential for a consistent interpretation of internal erosion test (Le et al, 2010).

\section{ACKNOWLEDGEMENT}

The authors wish to acknowledge Electicite de France (EDF-CIH) for the funding of the work and for their advice.

\section{BIBLIOGRAPHY}

Beltaos S., Rajaratnam. N. (1974). "Impinging circular turbulent jets." Journal of the Hydraulics Division, HY10: 1313-1328.

Foster, M., Fell, R. and Spannagle, M. (2000). "The statistics of embankment dam failures and accidents." Canadian Geotechnical Journal, 37, 1000-1024.

Hanson G.J, Simon A. (2001). "Erodibility of cohesive streambeds in the loess area of the Midwestern USA." Hydrological Processes, 15 (1): 23-38.

Hanson G. J., Cook K. R.(2004). "Apparatus, Tests procedures, and analytical methods to measure soil erodibility in-situ." Applied engineering in Agriculture, 20 (4): 455-462.

Le V.D., Marot D., Thorel L., Garnier J., Audrain P. (2010) "Centrifuge modelling of an internal erosion mechanism." 5th International Conference on Scour and Erosion (ISCE-5), 7-10 Nov, San Francisco, in press.

Regazzoni P.-L., Marot D., Wahl T., Hanson G. J., Courivaud J.-R. (2008). "Soils erodibility: a comparison between the Jet Erosion Test and the Hole Erosion Test." Proc. Inaugural International Mechanics Institute (EM08), Conference (A.S.C.E.), 18-21 May, Minneapolis, USA.

Regazzoni P.-L. (2009). Confrontation et analyse d'érodimètres et caractérisation de la sensibilité à l'érosion d'interface. $P h D$ thesis, Université de Nantes, $155 \mathrm{p}$.

USBR, United States Department of the Interior Bureau of Reclamations (1987). "Design of small dams." Water Resources Technical Publication. 